\title{
Aspect Clinique Atypique De La Tuberculose Oculaire A Propos D'un Cas
}

\author{
Samia Nassik \\ Aouatef Masmoudi \\ Salssabile Nafizy \\ Saad Mouine \\ Amine Kouisbahi \\ Amina Berraho
}

Service d'Ophtalmologie B. Hôpital des Spécialités, Chu IBN Sina Rabat, Quartier Souissi 6220 Rabat, Maroc

Doi: 10.19044/esj.2018.v14n9p122 URL:http://dx.doi.org/10.19044/esj.2018.v14n9p122

\begin{abstract}
Introduction: The ophthalmological manifestation of tuberculosis varies significantly, and almost all the elements of the globe and orbit can be reached. Methods and Results: The aim of this work was to report a clinical aspect of ocular tuberculosis in a young woman who has a notion of tuberculous contagion and who was presented at the fundus of a macular papillary membrane with a tubercle of Bouchut or papillary tubercle. The diagnosis was retained on a bundle of clinical, biological, and radiological arguments. The evolution under treatment was favorable with an improvement of its visual acuity. Conclusion: It is necessary to know how to evoke the tuberculous etiology in view of all ocular inflammation, even atypical. This is required considering the great polymorphism of its clinical characteristics.
\end{abstract}

Keywords: Ocular tuberculosis, epi macular membrane, anti-tuberculosis treatment

\section{Résumé}

Introduction: Les manifestations ophtalmologiques de la tuberculose sont très variées ; presque tous les éléments du globe et de l'orbite peuvent en être atteints. Matériel et résultat: Le but de ce travail a été de rapporter un aspect clinique de la tuberculose oculaire chez une jeune femme qui a une notion de contage tuberculeux et qui a présenté au fond d'œil une membrane tractionnelle papillo maculaire avec un tubercule de Bouchut sous papillaire. Le diagnostic a était retenu sur un faisceau d'arguments cliniques, biologiques 
et radiologiques. L'évolution sous traitement était favorable avec une amélioration de son acuité visuelle. Conclusion: Il faut savoir évoquer l'étiologie tuberculeuse face à toute inflammation oculaire même atypique. Cela est requis vu le grand polymorphisme de ses tableaux cliniques.

Mots-clés: Tuberculose oculaire, membrane épi maculaire, traitement antituberculeux

\section{Introduction}

La tuberculose est une infection grave. Elle sévit à l'état endémique dans les pays en voie de développement. Sa recrudescence avec l'avènement du SIDA et l'apparition de souches multi résistantes font actuellement de cette maladie un problème de santé publique dans les pays développés. La localisation intra-oculaire de la tuberculose est rare. Elle est observée essentiellement dans les formes miliaires.

\section{Patient et Observation}

Nous rapportons un cas d'une jeune femme 36 ans aux antécédents de contage tuberculeux ; elle se plaint d'une baisse rapidement progressive de l'acuité visuelle (AV) de son œil droit. A l'examen ophtalmologique l' AV de l'œil droit est à compter les doigts à $3 \mathrm{~m}$; le segment antérieur est normal; le fond d'œil montre une membrane papillo-maculaire faisant traction sur la macula et réalisant un nœud, un nodule bien circonscrit blanchâtre sous papillaire dans l'aire temporal supérieur, des vaisseaux grêles et tortueux. La patiente a bénéficié d'un bilan ophtalmologique complet, d'un bilan biologique et d'un bilan radiologique qui ont permis d'établir le diagnostic de tuberculose oculaire. L'examen de l'œil gauche est strictement normal avec une acuité visuelle de 10/10. En collaboration avec le service de pneumophtisiologie notre patiente a été mise sous antibacillaires associés aux corticoïdes par voie générale. L'acuité visuelle s'est améliorée à $3 / 10$ faible avec même aspect au fond d'œil. Une chirurgie endo-oculaire a été proposée à la patiente pour pelage de la membrane épimaculaire et éviter ainsi un décollement de rétine tractionnel. 


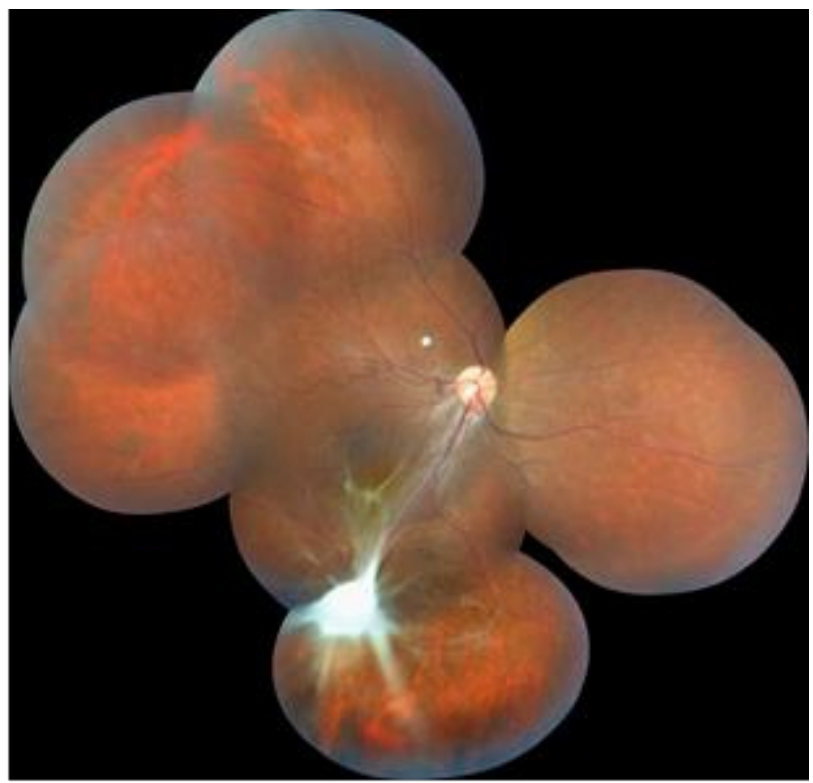

Figure 1. Rétinophotographie OG. Membrane papillo-maculaire réalisant un noeud avec une formation blanchâtre à contour net, plane, sous papillaire

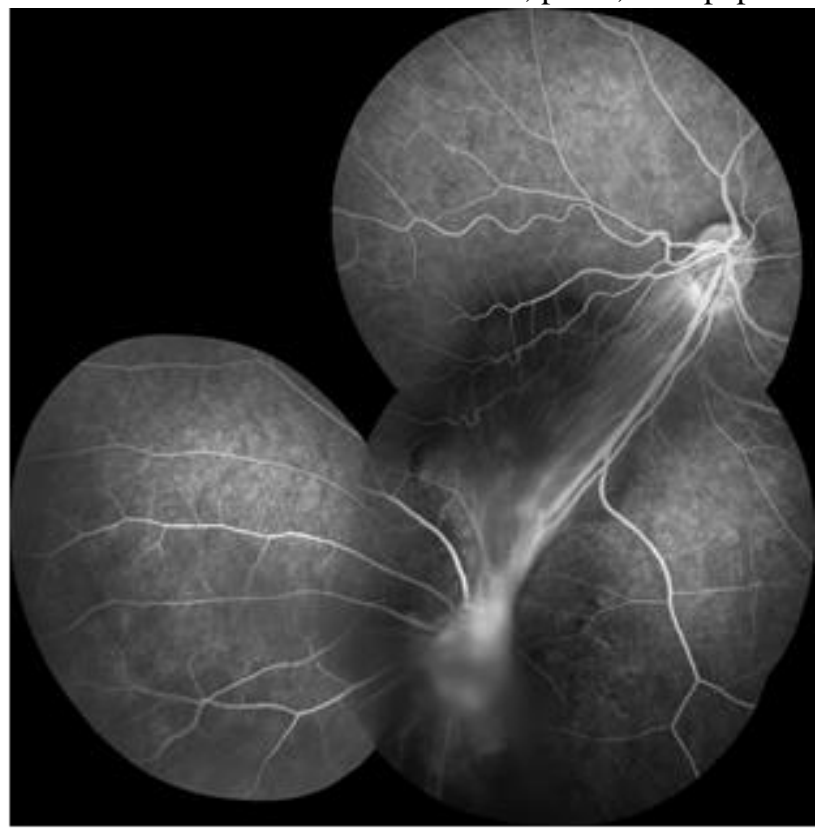

Figure 2. Angiographie à la fluorescéine. Membrane papillo- maculaire mimant un décollement de rétine tractionnel

\section{Discussion}

La tuberculose est une cause majeure de morbi-mortalité dans les pays en voie de développement. La prévalence de la tuberculose dans le monde est estimée à environ 10 millions de nouveaux cas par an. Au Maroc on recense 
environ 25 milles nouveaux cas / an (Laktaoui et al., 2009; Karim et al., 2003). Les localisations extra-pulmonaires représentent 35\% de l'ensemble des atteintes, dont 1 à $2 \%$ sont des atteintes oculaires (Benchekroun et al., 1999; El Bakkali et al., 2001). Les atteintes ophtalmologiques liées à la tuberculose sont diverses: toutes les structures de l'œil, à l'exception du cristallin, peuvent être concernées. Certaines de ces manifestations, comme les tuberculomes ou les tubercules de Bouchut, sont liées à la présence du bacille dans 1'œil; d'autres, parmi lesquelles certaines formes d'uvéites, sont le reflet d'une hypersensibilité retardée au bacille de Koch (El Bakkali et al., 2001).

Le traitement antituberculeux standard est bien codifié par des recommandations récentes et débute par une quadrithérapie d'induction associant isoniazide, rifampicine, éthambutol et pyrazinamide pendant 2 mois. Une bithérapie de consolidation associant isoniazide et rifampicine y fait suite, pour laquelle une durée de 4 mois semble habituellement suffisante bien qu'une durée de 7 à 10 mois est souvent recommandée par précaution. Plusieurs essais cliniques montrent que la corticothérapie adjuvante doit être systématique pour 8 semaines dont 4 à fortes doses (Akhaddar et al., 2001). Un traitement symptomatique spécifique doit être mis en place en cas de complications générales, notamment d'hyponatrémie. La surveillance de la tolérance et de l'efficacité du traitement est essentiellement clinique.

\section{Conclusion}

La tuberculose oculaire reste une entité rare, celle-ci peut constituer une circonstance de découverte de la maladie et peut rester isolée sans aucune association avec une atteinte pulmonaire. La difficulté du diagnostic est due au polymorphisme des signes oculaires. Son diagnostic est, dans la plupart du temps, présumé en se basant sur un faisceau d'argument clinique et biologique. Le pronostic visuel dépend de l'instauration d'un traitement adapté permettant une stabilisation ou une amélioration visuelle.

\section{Déclaration d'intérêt}

Les auteurs déclarent ne pas avoir de conflit d'intérêt en relation avec cet article.

\section{Contributions des auteurs}

Tous les auteurs ont participé à la réalisation de l'article. Tous les auteurs ont lu et approuvé la version finale du manuscrit.

\section{References:}

1. Akhaddar, A., El Hassani, MY., Chakir, N., \& Jiddane, M. (2001). Tuberculome optochiasmatique complication d'une méningite 
tuberculeuse: À propos d'un cas et revue de la littérature. J Neuroradiol. 28(2):137-42.

2. Benchekroun, S., El Mansouri, Y., Rachid, R., El Belhadji, M., Laouissi, N., \& Zaghloul, K. (1999). Granulome choroïdien pseudotumoral au cours de la tuberculose miliaire. J Fr Ophtalmol. 22(7):771-5.

3. El Bakkali, M., Halhal, M., Chefchaouni, M., Belmekki, M., Abdellah, H., \& Berraho, A. (2001) Uvéite tuberculeuse. J Fr Ophtalmol. 24(4):396-9.

4. Karim, A., Laghmari, M., Boutimzine, N., Lamarti, A., Ibrahimy, W., \& Essakali, N. (2003). Granulome choroüdien pseudotumoral révélant une tuberculose: à propos d'une observation. J Fr Ophtalmol. 26(6):614-7.

5. Laktaoui A, et al. (2009). Tuberculome maculaire et névrite optique: association rare au cours d'une méningo-encéphalite tuberculeuse. $\mathrm{J}$ Fr Ophtalmol. Nov;32(9):673-8. [PubMed]. 